\title{
The research and implementation for the conjugate vice type line of stator and rotor of single screw motor
}

\author{
CHEN Xin ${ }^{1, a}$, CHEN Qi $i^{2, b}$ \\ ${ }^{1}$ Shenyang university of technology school of science, China \\ ${ }^{2}$ Shenyang university of technology school of science, China \\ a491079175@qq.com, bchenqi313520@126.com
}

Keywords: single-screw motor; stator; rotor; conjugate vice

\begin{abstract}
Because the stator of rubber shrinkage for single-screw motor in the manufacturing process is different, it leads to the need to study the matching of the rotor. This paper analyzes the screw motor line design method, according to meshing theory for the motion of the stator and rotor, numerical mesh calculation method and the knowledge of the screw drill, giving a method that is using of screw stator, rotor end contour of discrete data points to solve the numerical solution of conjugate vice for sub-curve. This method is achieved on the computer, verify the generated curve meshing of the stator and rotor, and it has certain application value and universality in engineering practice.
\end{abstract}

\section{Introduction}

Screw drill, also known as positive displacement motor, abbreviated as PDM, is a positive displacement downhole drill motor. Single screw motor is the volume of using of drilling fluids such as mud driven power machine. It has only two components, namely the rotor and stator. In the actual project, after the formation of the stator, cooling, because of the different of shrinkage, it leads to a matching rotor; when the production of a number does not match the rotor, it needs to find a matching stator and require the numerical simulation to solve such problems.

At present, screw motor performance is better that is produced in the United States, Russia and other countries, its speed range is big and it leaks little. China has the disparity away from it, but its key technologies will be not disclosed. So aiming to this problem, in order to improve the operating characteristics of the line of the analysis of screw motor design level and screw drill, and to shorten the gap between China and the advanced international level, we must be multi-pronged approach from the design, manufacture [1]. As far as the design is concerned, we do not know the equation of meshing abroad, but our measurement techniques can more easily measure the stator and rotor parameters, so this paper makes use of reverse engineering, basing on the meshing theory and numerical meshing calculation method and the knowledge of the screw drill, gives the algorithm that the stator and rotor respectively generates conjugate curve, generates mesh analysis on the computer, and develops a set rotor mutual conversion process.

\section{Meshing Theory}

In practice, with the different work conditions, the head of screw motor needed is different, the more head, the bigger torque and the less speed. Due to the different screw head and shapes, so the type line equation is different. Even if it is to achieve the same function in the same head situation. Because it involves a different method, screw type line is also different. Based on the principle of the motor movement, the rotor within the stator plane motions of the planets, the movement can be decomposed into two, the one is rotor rotation movement around its own center $O_{1}$ of gyration, the direction is clockwise, the other is the revolution of the rotor around the stator bushing center $O$ movement, the direction is counterclockwise, as shown in Figure 1, it is a screw motor principle 
diagram, in the Figure $\alpha$ is the angle of rotation of the rotary center $O_{1}$ of the rotor itself, and $\beta$ is the angle of revolution of the rotor bushing center $O$.

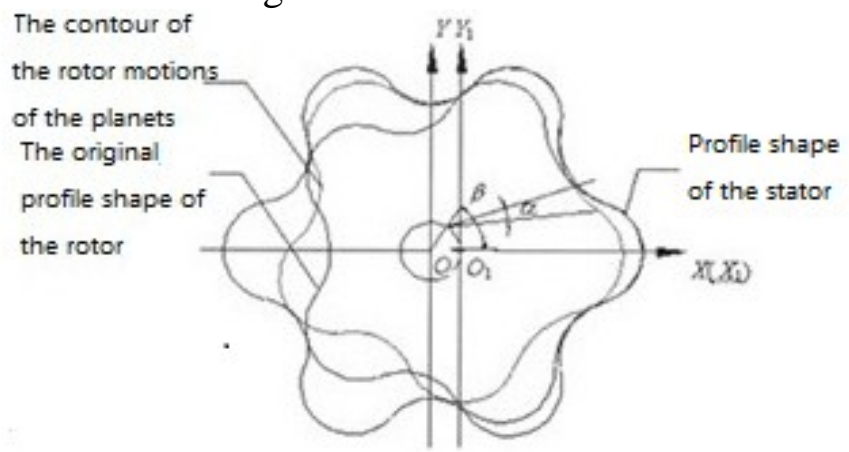

Figure 1 schematic diagram of the screw motor sports

The yoke vice of rotor and stator for screw drill needs to meet the isolation conditions and the disappearance of conditions [2], that is, when the rotor is in the any position of stator, Rotor and stator lines have number of contact points, the flow area (That is the cross-sectional area of the stator hole in the rotor that is not occupy all the parts of the area) is separated into several non-connected regions, called the isolation conditions. Under the process of planetary motion of rotor linear in the stator linear. Due to the change in the contact point, in each region of the location, shape, area have to change, some areas should be larger, some smaller. The conditions that the line type must have are: the regional area can be smaller zero; this condition is known as the disappearance of the conditions of the region [3].

If the stator is fixed, the rotor can do the motions of the planets in the stator plane. Secondly, it also needs to meet the conditions of isolation and the disappearance of the conditions described above. Calculation by the program, we can calculate the meshing point of the rotor and stator mesh with each other at any moment, the use of more accurate interpolation can be obtained in the numerical analysis mesh points. In theory, such as the head of $N$ rotor, the mesh of rotor and stator has $N$ fixed contact point and a mobile contact point. If the results of the program calculating meet this conclusion and the method of mesh points to the distance difference is small (the accuracy is $0.001[\mathrm{~mm}]$ ) and uniform, so we can consider that the total yoke vice is ideal $[4,5]$.

\section{Generation Algorithm of the Conjugate Curves}

\section{Algorithm of rotor generating to stator.}

(1)To set up the half-cycle of data and transformation, it generates a cycle of data, and then it applies a similar reason through mathematical transformation using a data cycle to calculate the full cycle of the rotor profile shape data.

(2)Based on meshing theory for the motion of the rotor and numerical calculation method, the profile shape data of stator can be getted.

Specifically, the following:

Figure 2 shows the rotor of a cycle graph, assuming that the rectangular coordinates of the half cycle at any point on the rotor $(x[i], y[i])$, and corresponds to the next half cycle data $(X[i], Y[i])$ is:

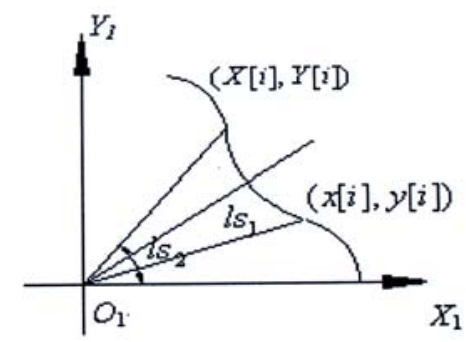

Figure 2 rotor solving figure of second half cycle 
$\left\{\begin{array}{l}X[i]=\operatorname{sqrt}\left(l s_{1}\right) \cos \left(l s_{2}\right) \\ Y[i]=\operatorname{sqrt}\left(l s_{1}\right) \sin \left(l s_{2}\right)\end{array}\right.$

Among

$\left\{\begin{array}{l}l s_{1}=x[i] x[i]+y[i] y[i] \\ l s_{2}=2 \pi / 5-a \tan (y[i] / x[i])\end{array}\right.$

The formula: $l s_{1}$ - The corresponding polar radius of the profile shape data for the semi-cycle on the rotor;

$l s_{2}$ - The corresponding very angle of the profile shape data for the second half on the rotor.

Showing in Figure 1, setting the cartesian coordinate system $X O Y$ as the stator coordinate system, cartesian coordinate system $X_{1} O_{1} Y_{1}$ is the rotor coordinate system, generally rotor datas given are corresponding to the rotor coordinate system. Assume in the coordinate system $X_{1} O_{1} Y_{1}$, the original data coordinates for rotor profile shape at any point is $(X Z, Y Z)$, angle of revolution for rotor at a time is $\beta$, so at this point the corresponding angle of rotation is $\beta / N$ ( $N$ is the number of rotor head), In a mathematical operation, we set the counterclockwise angle is positive, clockwise negative, by the coordinate rotation formula getting the rotor rotation angle $\beta / N$ and revolution angle $\beta$, the profile shape data of rotor is:

$\left\{\begin{array}{l}X_{1}=X \cdot \cos (-\beta / N)-Z Z \sin (-\beta / N)-e+e \cos (\beta \\ Y_{1}=X \cdot \sin (-\beta / N)+Z \cdot \cos (-\beta / N)+e \sin (\beta)\end{array}\right.$

Making the datas we calculate form (3) converse to stator coordinate system, and we can get corresponding of data point coordinates for the stator profile:

$\left\{\begin{array}{l}X D=X_{1}+e \\ Y D=Y_{1}\end{array}\right.$

In formula (4): $e$ - the eccentric, the unit is [mm]; $N$-the number of rotor head.

Making Angle $\beta$ increasing from $0^{\circ}$ to $\nabla \beta$ Angle, getting $\nabla \beta=0.5^{\circ}$, it can form many envelope after plane motions of the planets that the rotor dose. When the angle $\beta$ changes to $2 \pi$, the rotor is back to the starting position and cycles, so we can only calculate the outer profile shape of all the envelope in $0 \sim 2 \pi$, and it can be considered the data on the stator profile shape.

\section{Algorithm of stator generating to rotor.}

(1)The same to the first step of the above algorithm, getting the profile shape data of the full cycle of the stator.

(2)Basing on the idea of above algorithm, applying the knowledge of the polar angle and polar radius, through profile shape data of stator backstepping profile shape data of rotor.

Specifically, the following:

The same to the above algorithm, we can get profile shape discrete data of the stator. In the appeal coordinate system, setting any point of coordinates for the rotor shaped profile at the zero position as $\left(X Z_{1}, Y Z_{1}\right)$, so basing on above algorithm, rotor profile shape data $\left(X Z_{2}, Y Z_{2}\right)$ can also be obtained, and then basing on the rotor shaped profile getting stator shaped profile $\left(X D_{1}, Y D_{1}\right)$. At a time what we know is datas of stator profile shape, and therefore deciding $\left(X D_{1}, Y D_{1}\right)$ based on the polar angle and polar radius at this time, and then reversing $\left(X Z_{1}, Y Z_{1}\right)$.After each turning an angle, we can also use it to determine the coordinates of the rotor, finally getting profile shape data of rotor.

Analysis of meshing.After data generated, we get the co-ordination of a rotor and stator data. several graphics of figure 3 is the case when the meshing of the rotor and stator in different locations, due to limited space, we can not display every movement that the rotor rotates in the stator, here only 
quoting the several representative graphics, when rotor turns one time, we can get several meshing points, this shows that meshing of screw's rotor and stator is good.

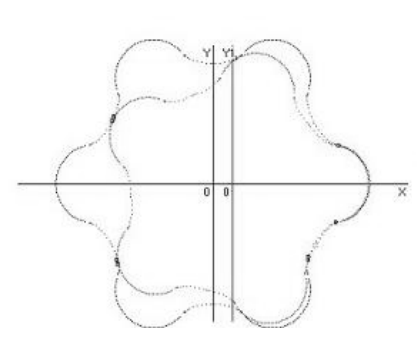

a) 0 degrees

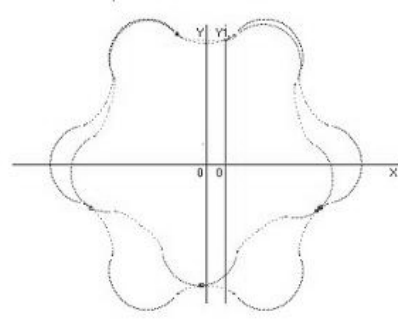

b) 10 degrees

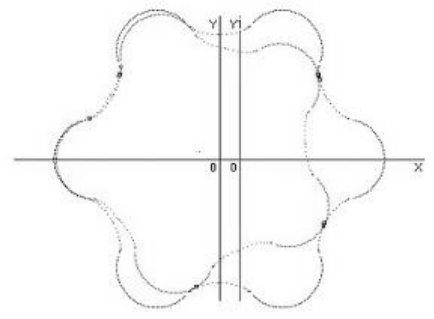

c) 100 degrees

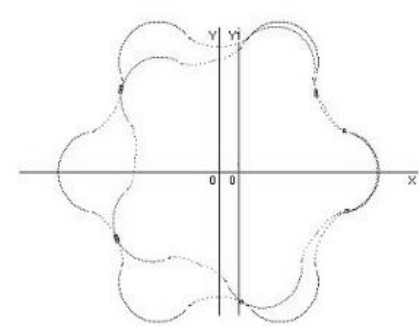

d) 180 degrees

Figure 3 the meshing of the rotor and stator in different locations

\section{Software Implementation}

In order to complete the stator and rotor data of the downhole drilling motor generated and the analysis for the meshing movement of stator and rotor. The paper carries a lot of program. The program is under the Windows XP operating system, application $\mathrm{C}++$ Builder 6.0 compiled. The program's main function is giving rotor profile shape data and stator profile shape data, based on Meshing Theory, realizing the transformation, generating the corresponding conjugate curves, and mimic motion process, giving mesh situation of different positions.

\section{Conclusions}

(1) By the numerical meshing approach on the design of the screw drill meshing pair is feasible in theory and practice. Numerical design method avoid the traditional design based on the analytical expressions for conjugate type line is difficult, adjust the convenient faults, can be faster and more common to complete the design, and design the type line of the meshing properties and strength verification, precision is high, can meet the practical demands.

(2) By the numerical method to analyze the meshing feature of meshing pair is useful. According to the conditions that need to meet of screw motor meshing, this paper further analyzes mesh point of contact law for rotor and stator of screw motor, giving a method that makes use of numerical meshing to characteristics analysis, observing visually meshing process, meeting the meshing theory, and providing practical use for the reasonable reference.

\section{References}

[1] Su Yiniao, Xie Huahuang. Linear analysis and research method of linear forscrew motor [J]. Oil Drilling Machines, 1985, (6): 12-16.

[2] Wang Jiezhong, Yi Xianzhong, Wu Guisheng. Conjugate linear analysis for single head single screw water conservancy machine [J].Journal of Petroleum Machinery. 1997, 7 (25):1-100.

[3] Su Yinao. The research and application of screw drill [J]. Beijing: Press of Oil Industry, 2000.

[4] Yu Lianjiang. Screw motor of positive displacement [J] Machinery Manufacturing, 1994(11):4-5.

[5] Guo Changsheng, Tang Yan.Screw Rotor Profile Manufacturability [J].Machining Science and Technology.2003, 7(1):53-64. 